\title{
Barrier-to-Autointegration Factor (BAF) involvement in prelamin A-related chromatin organization changes
}

\author{
Manuela Loi ${ }^{1,2}$, Vittoria Cenni ${ }^{1,2}$, Serena Duchi ${ }^{3,4}$, Stefano Squarzoni ${ }^{1,2}$, \\ Lopez-Otin ${ }^{5}$, Roland Foisner ${ }^{6}$, Giovanna Lattanzi ${ }^{1,2}$, Cristina Capanni ${ }^{1,2}$ \\ ${ }^{1}$ CNR-National Research Council of Italy, Institute of Molecular Genetics, Unit of Bologna, 40136 Bologna, Italy \\ ${ }^{2}$ Laboratory of Musculoskeletal Cell Biology, IOR, 40136 Bologna, Italy \\ ${ }^{3}$ Osteoarticolar Regeneration Laboratory, Rizzoli Orthopaedic Institute, 40136 Bologna, Italy \\ ${ }^{4}$ Department of Biomedical and Neuromotor Sciences (DIBINEM), University of Bologna, 40123 Bologna, Italy \\ ${ }^{5}$ Department of Biochemistry and Molecular Biology, Medical Faculty, Oviedo University, 33006 Oviedo, Spain \\ ${ }^{6}$ Max F. Perutz Laboratories, Medical University of Vienna, A-1030 Vienna, Austria
}

Correspondence to: Cristina Capanni, e-mail: ccapanni@area.bo.cnr.it

Keywords: prelamin A, barrier-to-autointegration factor, chromatin, Hutchinson-Gilford progeria syndrome, Nestor-Guillermo progeria

Received: August 25, 2015

Accepted: November 21, 2015

Published: December 20, 2015

\section{ABSTRACT}

Chromatin disorganization is one of the major alterations linked to prelamin A processing impairment. In this study we demonstrate that BAF is necessary to modulate prelamin A effects on chromatin structure. We show that when prelamin A and BAF cannot properly interact no prelamin A-dependent effects on chromatin occur; similar to what is observed in human Nestor Guillermo Progeria Syndrome cells harboring a BAF mutation, in HEK293 cells expressing a BAF mutant unable to bind prelamin A, or in siRNA mediated BAF-depleted HEK293 cells expressing prelamin A. BAF is necessary to induce histone trimethyl-H3K9 as well as HP1-alpha and LAP2-alpha nuclear relocalization in response to prelamin $A$ accumulation. These findings are enforced by electron microscopy evaluations showing how the prelamin A-BAF interaction governs overall chromatin organization. Finally, we demonstrate that the LAP2-alpha nuclear localization defect observed in HGPS cells involves the progerin-BAF interaction, thus establishing a functional link between BAF and prelamin A pathological forms.

\section{INTRODUCTION}

BAF is a DNA binding protein that allows DNA filament interconnection in vivo and condensing of longer DNA molecules in vitro [1]. BAF localizes ubiquitously in cells, and several nuclear physiological events including post-mitotic nuclear assembly, chromatin remodeling, gene expression and DNA damage repair, seem to depend on proper BAF cellular distribution and expression [2], [3]. In the nucleus, BAF directly binds three fundamental groups of proteins: LEM-domain proteins [4-7], histones [8], [9] and nuclear lamins [10], [5]. Lamins are components of the nuclear lamina, a proteinaceous meshwork underlying the inner nuclear membrane. This structure arises from the polymerization of type $\mathrm{V}$ intermediate filaments encoded by the $L M N A$ gene, named lamin $\mathrm{A}$ and lamin $\mathrm{C}$, which, in combination with lamin B, provide a molecular link between the inner nuclear membrane and the genome. In particular, it has been demonstrated that components of the nuclear lamina directly interact with DNA and with proteins able to influence the accessibility to genetic information [11]. Thus, it is not surprising that a wide range of rare diseases, collectively named laminopathies, results from LMNA mutations. Muscular dystrophy, cardiomyopathy, neuropathy, lipodystrophy and progeroid syndromes are overlapping disorders identified in laminopathic patients [12]. At the molecular level, LMNA gene mutations affecting prelamin A processing lead to an acceleration in aging, causing adipose tissue atrophy, bone resorption and other systemic symptoms as described in Mandibuloacral Dysplasia (MAD), Hutchinson-Gilford Progeria Syndrome (HGPS), Familiar Partial Lipodystrophy type 2 (FPLD2) and Restrictive Dermophathy (RD) patients [12]. 
Prelamin A is the precursor of lamin A, and, unlike other types of lamins, it undergoes a specific maturation pathway. If maturation fails, prelamin A accumulation affects nuclear morphology [13], chromatin structure and DNA binding protein function [14-16] through a mechanism that is poorly understood.

We previously demonstrated in vivo molecular interaction between BAF and different prelamin A forms [17]. Prelamin A affects BAF cellular distribution inducing its nuclear localization; in accordance, prelamin A mutated forms identified in laminopathic cells have a similar effect [18]. Given that several chromatin modifying proteins have been identified among BAF binding partners [8], [9], [19], it is conceivable that the effects on chromatin organization caused by prelamin A could potentially depend on its interaction with BAF. The study reported here was aimed at demonstrating that the BAFprelamin A interaction is necessary to mediate prelamin A accumulation effects on chromatin organization. To this end, we took advantage of Nestor-Guillermo Progeria Syndrome (NGPS) skin fibroblasts induced to accumulate prelamin A, and HEK293 cells transfected with prelamin A constructs in combination with different BAF mutants. NGPS is a rare progeroid syndrome characterized by aged appearance, growth retardation and decreased subcutaneous fat [20]. This disease is due to a point mutation (c.34G $>$ A [p.Ala12Thr]) in the $B A N F 1$ gene, codifying for BAF. In our experiments we observed that the expression of both NGPS-BAF mutant and a BAF mutant (BAF-G47E) unable to interact with the inner nuclear membrane components, affect the ability of prelamin A to modify chromatin organization. We demonstrate that the redistribution of histone $\mathrm{H} 3$ trimethylated at lysine $9(\mathrm{H} 3 \mathrm{~K} 9 \mathrm{~m} 3)$, of HP1-alpha, and of the chromatin interacting protein LAP2-alpha, induced by prelamin A, need a proper prelamin A-BAF interaction. This is also required to preserve the overall prelamin A-dependent chromatin reorganization. In addition, we demonstrate the involvement of BAF in the deleterious effects triggered by progerin (a truncated prelamin A form accumulated in HGPS cells) on LAP2-alpha, observed in HGPS cells. Our results demonstrate a functional link between prelamin A and BAF allowing for a better understanding of the mechanism underlying pathological aging.

\section{RESULTS}

\section{NGPS cells show dysmorphic nuclei with altered BAF, lamin $\mathrm{A} / \mathrm{C}$ and prelamin A distribution which is associated with impaired prelamin A-mediated H3K9m3 intranuclear clustering}

In accordance with previously described results [21], we observed that in NGPS cells the BAF-A12T mutation affected BAF protein level. BAF was detectable in NGPS nuclei but hardly visible in the cytoplasm. In control cells, BAF was present in both cellular compartments (Figure 1A). Lamin A/C staining highlighted NGPS nuclear morphology defects, as described [21]. Increase in nuclear size and/or presence of nuclear blebs were observed in $80 \%$ of BANF1 mutated cells (Figure 1A asterisk and arrow, Figure 1B) while in control cells, less of $20 \%$ of nuclei were dysmorphic. Lamin A/C and BAF staining colocalized at the nuclear lamina of control and NGPS cells, however NGPS nuclei were characterized by honeycomb labeling patterns where rarefaction of Dapi staining was detectable (Figure 1C arrowhead).

In NGPS cells prelamin A processing is normal as demonstrated by the absence of prelamin A and progerin staining (Figure S1). Mevinolin addition to NGPS cells induced the accumulation of prelamin $\mathrm{A}$ at the nuclear lamina and at intranuclear aggregates, as observed in control cells. Prelamin A and BAF colocalized in control cells at both the nuclear periphery and the intranuclear aggregates (Figure 1D arrow and arrowhead, Figure 1E arrows). In contrast, in mevinolin-treated NGPS cells, though BAF nuclear recruitment was observed, BAFprelamin A colocalization was impaired. In particular we observed that colocalization at intranuclear aggregates was partially lost (Figure 1D-1E arrowheads) while, at the nuclear lamina both proteins revealed a colocalization characterized by honeycomb pattern (Figure 1D-1F arrow).

Prelamin A accumulation in mevinolin-treated cells induced $\mathrm{H} 3 \mathrm{~K} 9 \mathrm{~m} 3$ clustering in more than $80 \%$ of control cells in which the intranuclear aggregates of lamin A precursor perfectly colocalized with $\mathrm{H} 3 \mathrm{~K} 9 \mathrm{~m} 3$ aggregates (Figure 2A-2B, 2E and 2D arrow). On the contrary, in NGPS cells, even if prelamin A was accumulated and normally distributed in the nucleus, $\mathrm{H} 3 \mathrm{k} 9 \mathrm{~m} 3$ clustering failed (Figure 2B and 2C) and only a limited number of prelamin A intranuclear aggregates (50\%) colocalized with $\mathrm{H} 3 \mathrm{k} 9 \mathrm{~m} 3$ aggregates (Figure 2D arrowhead and 2E). However, it is important to note that in NGPS untreated cells, H3K9m3 was barely detectable and less than $20 \%$ of nuclei showed a normal clustered distribution, while in control cells a normal pattern was detectable in $60 \%$ of cells (Figure 2D and 2C).

\section{Prelamin A-BAF interaction is compromised by the $B A N F 1$ gene mutation occurring in NGPS cells}

Since in NGPS cells prelamin A and BAF colocalization appears partially lost, we wondered if prelamin A-BAF binding could be compromised by the BAF-A12T mutation. To this aim, HEK293 cells were transfected with FLAG-tagged lamin A (LA-WT) or FLAG-tagged prelamin A (LA-C661M) in combination with three GFP-tagged BAF constructs: wild type BAF (BAF-WT), NGPS-BAF mutant (BAF-A12T) and a BAF 


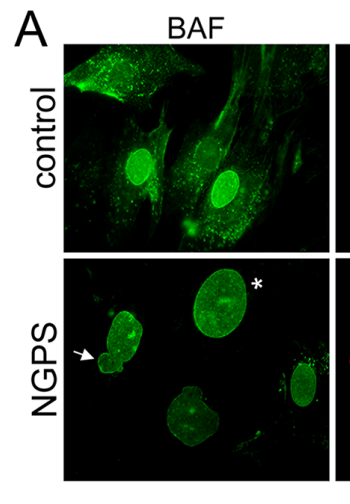

B

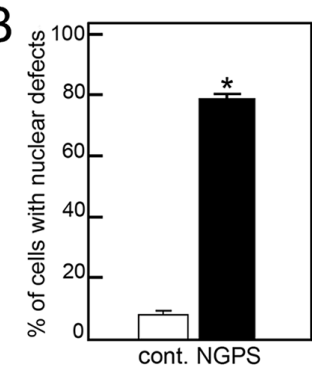

$\operatorname{lamin} \mathrm{A} / \mathrm{C}$
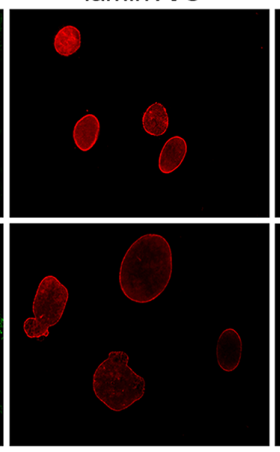

C

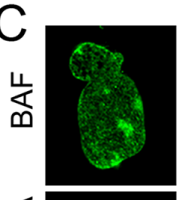

DAPI
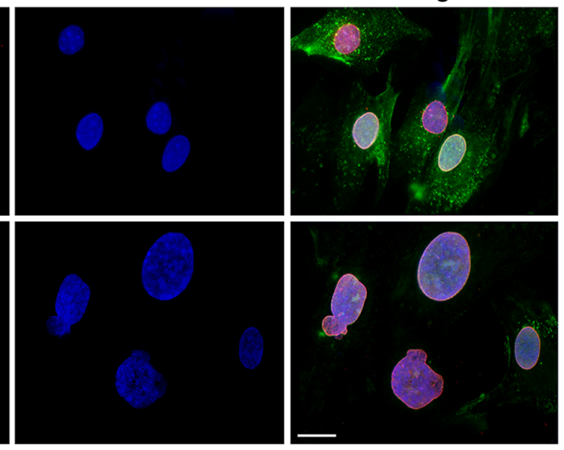

믐

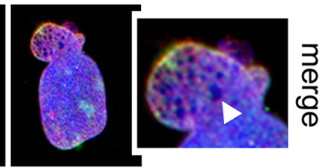

$\mathrm{D}$

prelamin A
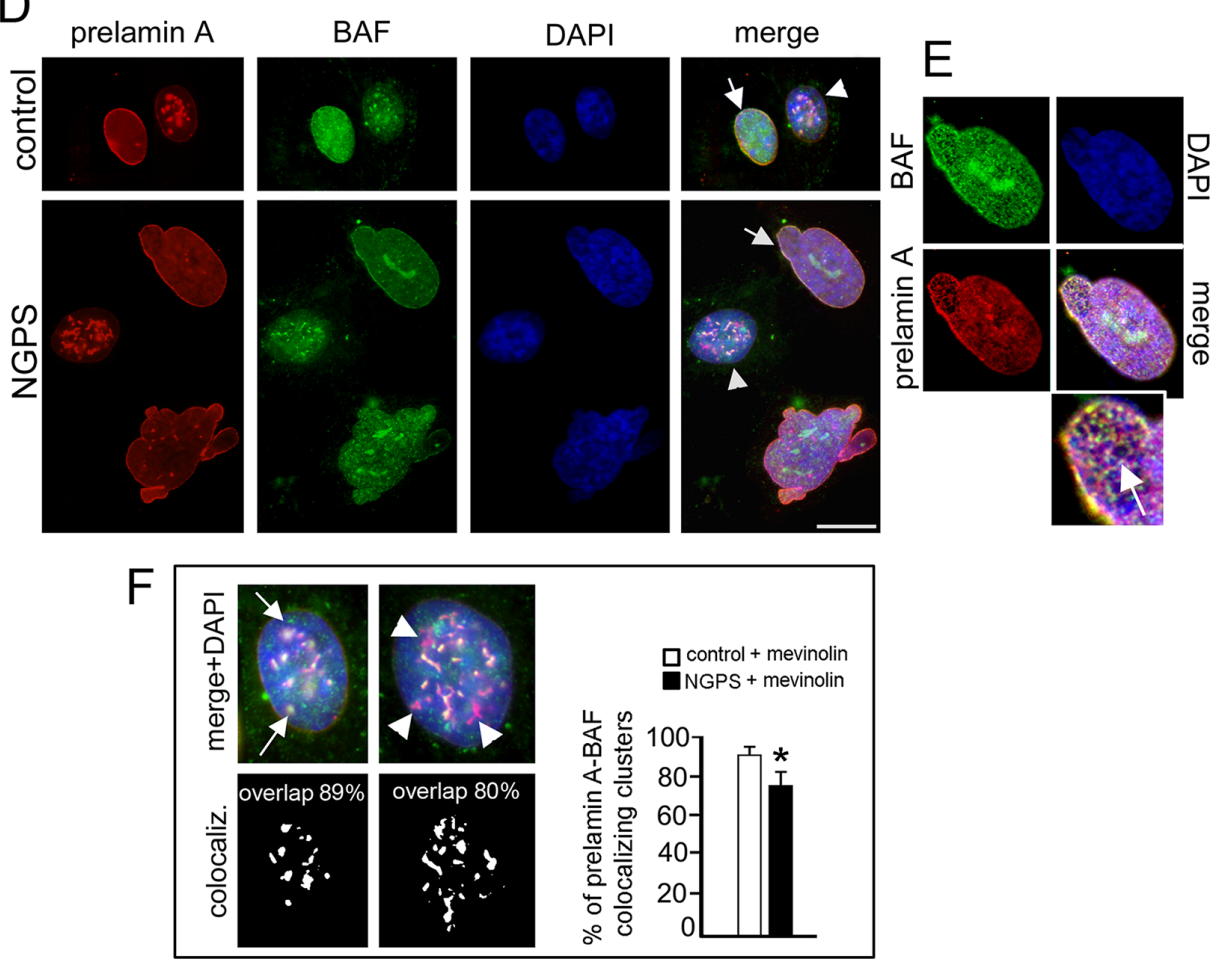

Figure 1: NGPS BAF mutation affects nuclear morphology, induces lamin A/C and prelamin A distribution defects. (A) BAF (green) and lamin A/C (red) localization in control and Nestor-Guillermo Progeria Syndrome (NGPS) cells. Asterisk: nuclear enlargement, arrow: nuclear bleb. Nuclei are costained by DAPI. Bar $10 \mu \mathrm{m}$. (B) The percentage of cells showing nuclear alterations is reported in the graph as means \pm S.D. of three different counts ( 100 nuclei per count). Asterisk: statistically significant difference at the Student's $t$-test $(P<0.05)$. (C) Higher magnification of NGPS nucleus indicated by arrow in A. Arrowhead: honeycomb. (D) Prelamin A (red) and BAF (green) immunofluorescence detection in control (cont.) and NGPS mevinolin treated cells. Arrows: prelamin A/BAF colocalization at the nuclear periphery. Arrowheads: nuclei with intranuclear prelamin A aggregates. DNA was stained by DAPI. Bar $10 \mu \mathrm{m}$. (E) Higher magnification of control (cont.) and NGPS nuclei with prelamin A intranuclear aggregates indicated by arrowheads in D. In a control nucleus prelamin $\mathrm{A} / \mathrm{BAF}$ costained intranuclear aggregates are indicated by arrows while in NGPS nucleus prelamin A intranuclear aggregates missing BAF staining are indicated by arrowheads. Percentage of prelamin A/BAF overlapped aggregates is indicated. The percentages of prelamin A foci colocalized with BAF foci are indicated. At least 100 cells were analyzed for each experiment. Data are presented as mean \pm S.D. $\left({ }^{*} P<0.001\right)$. (F) Higher magnification of NGPS cell nucleus indicated by arrow in D. Arrow: honeycomb structures. 
mutant unable to interact with nuclear envelope proteins (BAF-G47E) [17], [18], [8], [22]. Since, we previously demonstrated, in accordance with other authors [23] and [24], that the overexpression of LA-WT causes not only lamin A increase but also a definite level of prelamin A accumulation [17, 18], we harvested transfected cells 24 hour post-transfection. In this way, prelamin A protein amount in LA-WT expressing cells is lower than lamin A [17], [18].

The localization and expression of GFP-tagged proteins were evaluated (Figure 3A and Figure S2A). BAFWT and BAF-A12T showed a similar cytoplasmic/nuclear distribution while BAF-G47E localized exclusively in the nucleoplasm (Figure 3A arrow). The expression of BAFWT in combination with LA-WT or LA-C661M induced BAF-WT nuclear recruitment (Figure 3B-3C arrowheads) [17]. On the contrary, BAF-A12T mutant localization was slightly affected by expression of FLAG-tagged lamins. In particular, cytoplasmic GFP-BAF-A12T staining was still detectable (Figure 3B-3C arrows and Figure S4) and the nuclear rim signal increase was very low. As expected, BAF-G47E did not change at all its nucleoplasmic distribution (Figure 3B-3C and Figure S4). The analysis of the fluorescence intensity profile confirmed BAF-A12T and BAF-G47E translocation defects (Figure S4). Western blotting analysis showed that exogenous proteins were expressed at similar levels and at the predicted molecular weight (Figure S2A).

Anti-FLAG immunoprecipitation experiments performed in cotransfected cells showed a minor BAFA12T interaction with both LA-WT and LA-C661M compared with BAF-WT recovery. As expected, a faint band corresponding to BAF-G47E was observed only after a long exposure time (Figure 3D).

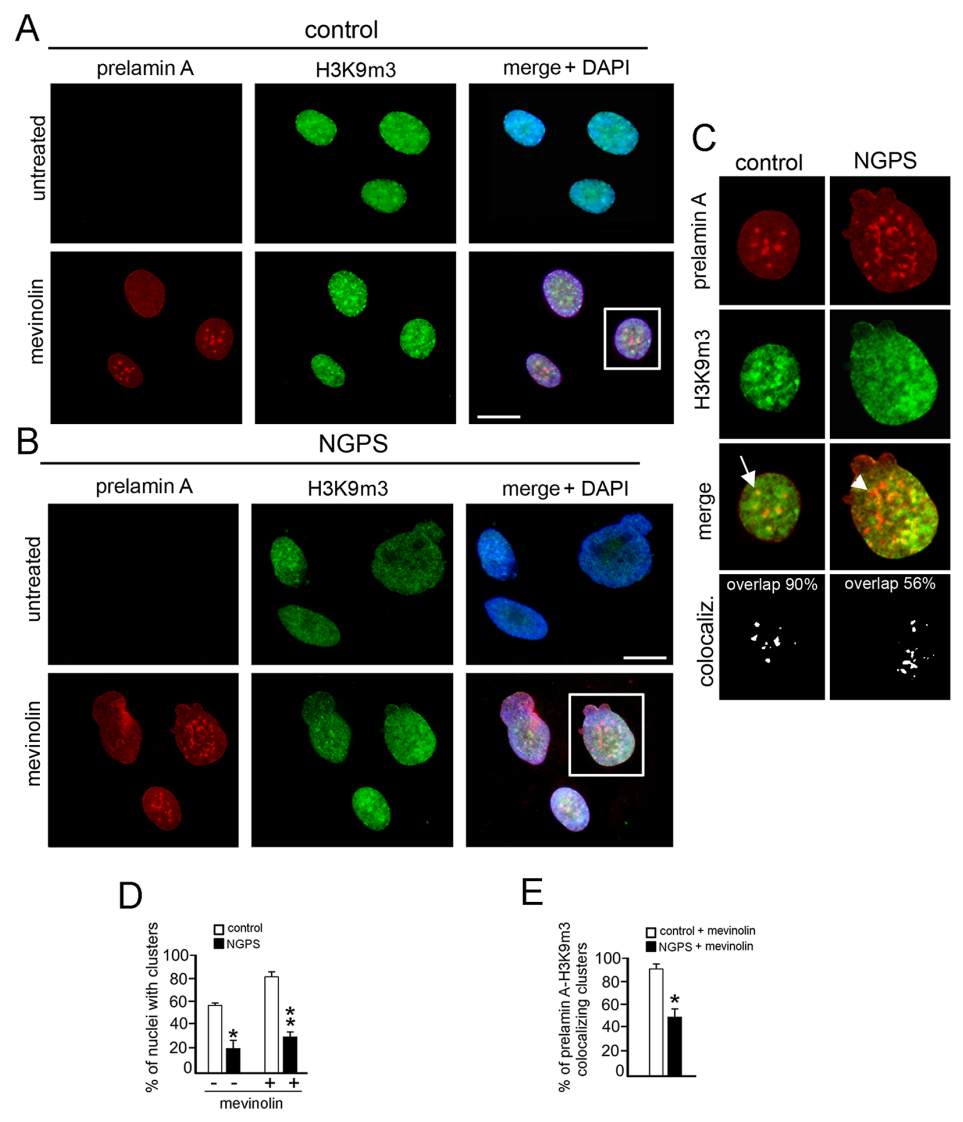

Figure 2: NGPS BAF mutation impairs prelamin A-related H3K9m3 intranuclear clustering. (A) Prelamin A (red) and histone $\mathrm{H} 3$ trimethylated at lysine 9 (H3K9m3, green) localization in control fibroblasts untreated or treated with mevinolin. DNA staining with DAPI is shown in merge (merge + DAPI). Bar $10 \mu \mathrm{M}$. (B) Prelamin A (red) and H3K9m3 (green) localization in NGPS fibroblasts untreated or treated with mevinolin. DNA staining with DAPI is shown in merge (merge + DAPI). Bar $10 \mu \mathrm{M}$. (C) Higher magnification of nuclei showed in A and B (squares). Prelamin A (red), histone H3K9m3 (green) and merge without DAPI staining are shown. Arrow: prelamin A-H3K9m3 costained intranuclear aggregate; arrowhead: prelamin A intranuclear aggregate in which $\mathrm{H} 3 \mathrm{~K} 9 \mathrm{~m} 3$ staining is missing. The percentage of prelamin A-H3K9m3 colocalized spots is reported. (D) Statistical analysis. The graph shows the percentage of nuclei showing clustered H3K9m3 in control and NGPS cells. White bars: control cells untreated (-) or mevinolin treated (+); black bars: NGPS cells untreated $(-)$ or mevinolin treated $(+)$. Values are means of two independent experiments \pm S.D.; for each experiment a total of 100 nuclei were considered. $\left({ }^{*} P<0.004\right),\left({ }^{* *} P<0.001\right)$. (E) The percentages of prelamin A foci colocalized with $\mathrm{H} 3 \mathrm{~K} 9 \mathrm{~m} 3$ are indicated. Values are means of two independent experiments \pm standard deviation. At least 100 cells were analyzed for each experiment. $(* P<0.001)$. 


\section{Prelamin A induces HP1-alpha and LAP2-alpha nuclear relocalization by interacting with $\mathrm{BAF}$}

Since the BAF mutation occurring in NGPS cells not only compromises prelamin A-BAF interaction but also impairs prelamin A-mediated $\mathrm{H} 3 \mathrm{~K} 9 \mathrm{~m} 3$ intranuclear recruitment, we wondered if this last alteration could also be caused by anomalous interaction of prelamin A and BAF. To this aim we evaluated the effect of prelamin A on chromatin marker distribution in models where prelamin $\mathrm{A}$ and BAF fail to interact properly.

The expression of prelamin $\mathrm{A}$ is reported to induce HP1-alpha clustering without any effects on protein level $[25,26]$. A similar result was obtained in HEK293 expressing prelamin A constructs (Figure S3). On the contrary, HP1-alpha localization was unaffected by the expression of GFP-empty vector or GFP-BAF constructs (Figure S2B and 4A).
In cells expressing prelamin A constructs (LA-WT and LA-C661M) in combination with BAF-WT, HP1alpha nuclear periphery recruitment was detectable (Figure 4A'-4A" and 4a'-4a" arrowheads) while in cells expressing prelamin A constructs in combination with BAF mutants, the effect on HP1-alpha was impaired (Figure 4A'-4A" arrows and 4a'-4a"). However, in LAC661M/BAF-A12T expressing cells, a barely detectable HP1-alpha staining was perceptible at the nuclear rim (Figure 4A"and a" asterisks). Western blotting analysis showed that this result was not related to protein amount or molecular weight changes (Figure S2B).

To strengthen our finding, we evaluated also LAP2alpha [25]. This nucleoplasmic protein interacts with intranuclear lamins, and the accumulation of prelamin A modifies its localization [25]. In cells expressing prelamin A constructs alone or in combination with BAF-WT, we observed that LAP2-alpha was located at the nuclear
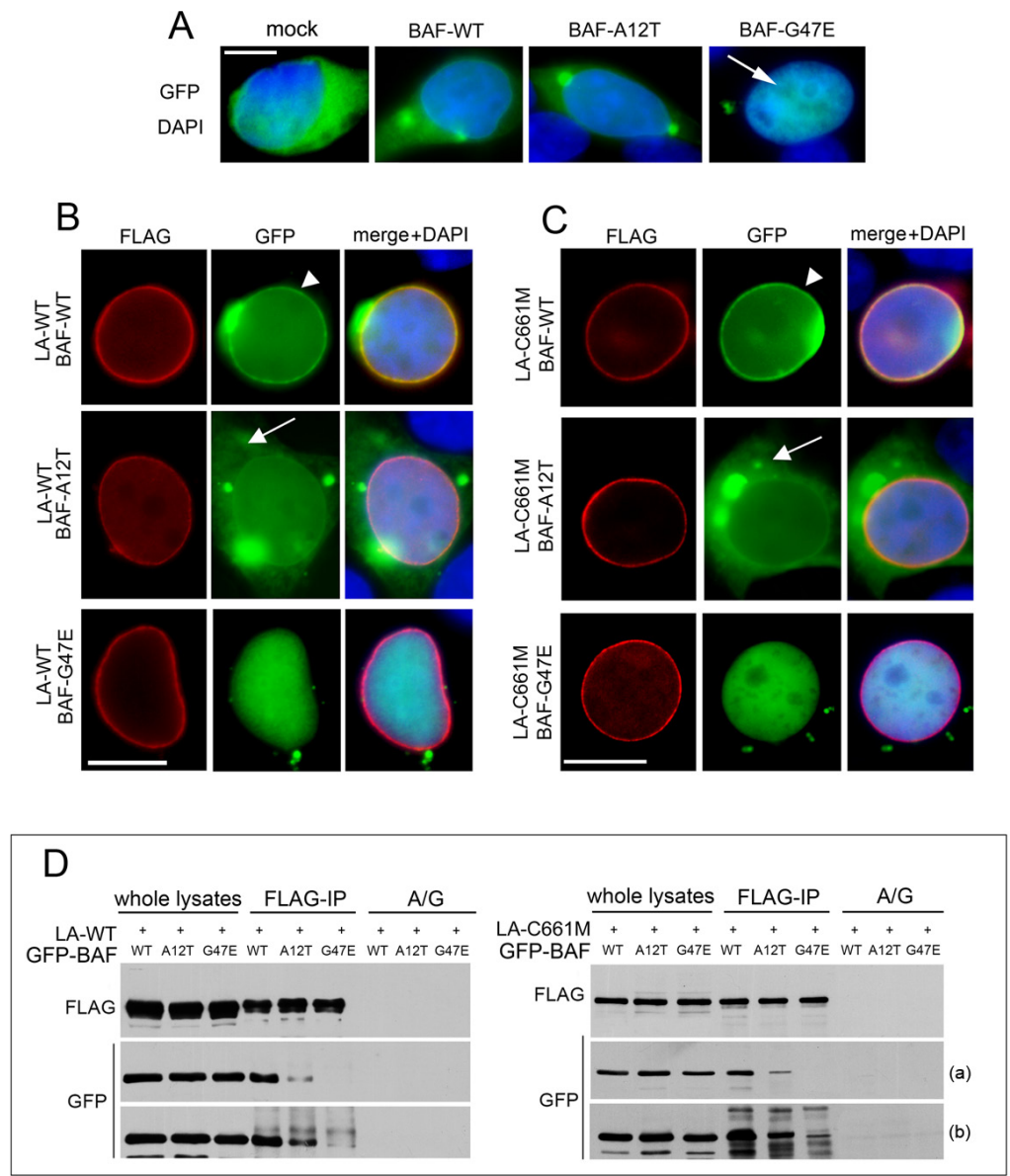

Figure 3: Evaluation of BAF mutants localization and interaction with prelamin A. (A) GFP-BAF mutants localization in HEK293 cells. GFP-empty vector (mock), BAF-WT, BAF-A12T and BAF-G47E localization in combination with DAPI staining is shown. Bar $10 \mu \mathrm{M}$. (B) GFP-BAF-mutants localization in FLAG-tagged wild type lamin A (LA-WT) expressing cells. (C) GFP-BAF-mutants localization in FLAG-tagged prelamin A (LA-C661M) expressing cells. In B and C FLAG staining (red), GFP signal (green). Arrowheads: nuclear lamina GFP localization; arrows: cytoplasmic GFP distribution. DAPI staining is shown in merge. Bar $10 \mu \mathrm{M}$. Images shown are representative of $80 \%$ of transfected cells. (D) Coimmunoprecipitation study performed in HEK293 cells transfected with LA-WT or LA-C661M in combination with GFP-BAF constructs. Western blotting analysis of FLAG and GFP-tagged proteins in whole lysate and coimmunoprecipitated complexes (FLAG-IP) are shown. No proteins staining was observed in absence of anti-FLAG antibody (A/G). Two different exposures, short (a) and long (b), are shown. 


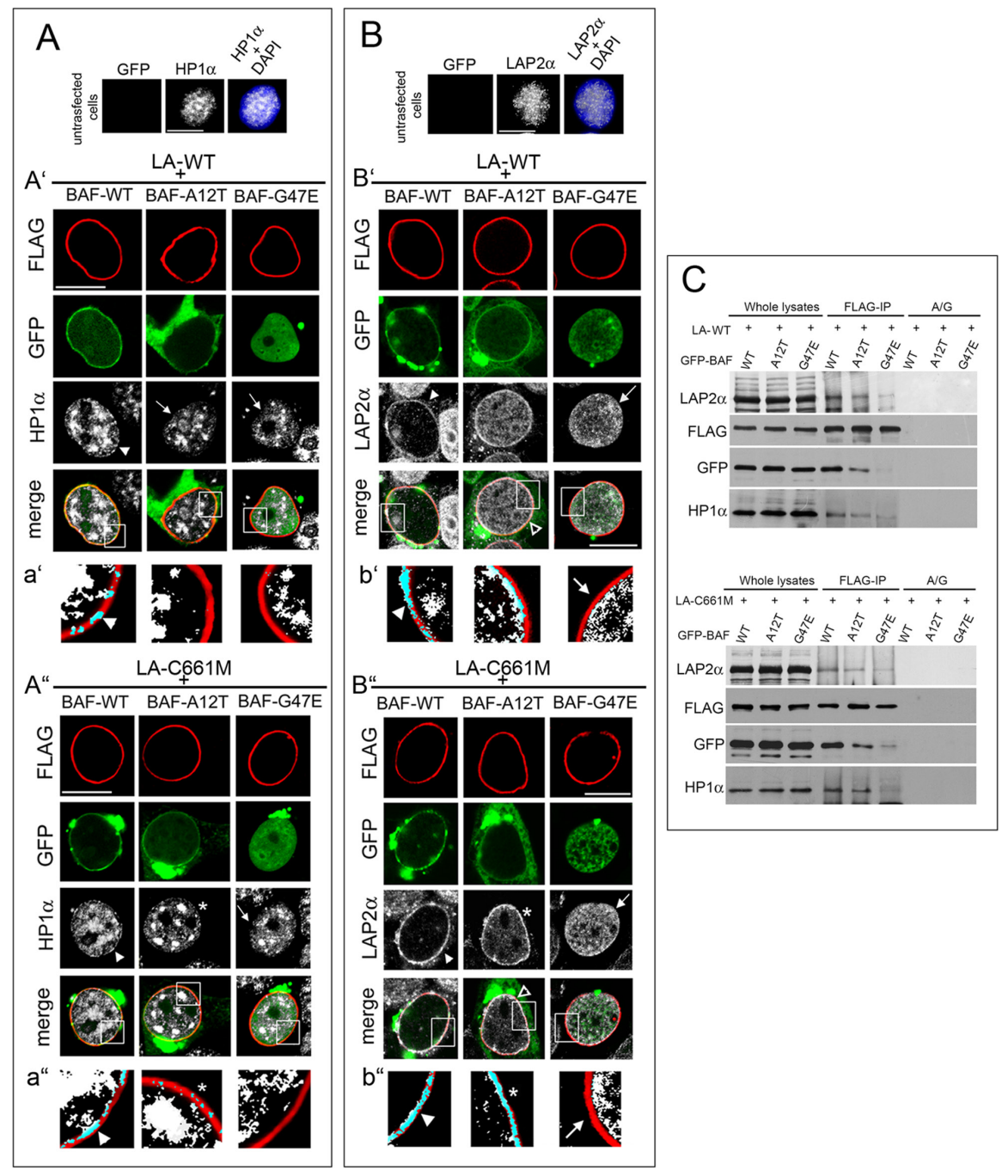

Figure 4: BAF governs HP1-alpha and LAP2-alpha nuclear relocalization in prelamin A accumulating cells. (A) HP1alpha $(\mathrm{HP} 1 \alpha)$ localization in HEK293 cells expressing FLAG-tagged lamin constructs in combination with GFP-BAF mutants. Top line: HP1 $\alpha$ nuclear distribution in untransfected cells. HP1 $\alpha$ staining (grey) is combined with DAPI (HP1 $\alpha+$ DAPI). Bar $10 \mu \mathrm{M}$. A') HP1 $\alpha$ localization in cells expressing wild type lamin A (LA-WT+) in combination with BAF-WT or BAF-A12T or BAF-G47E. FLAG staining (red), GFP (green), HP1 $\alpha$ (grey). Bar $10 \mu \mathrm{M}$. a') Density dot plot of FLAG and HP1 $\alpha$ colocalization staining showed in A'. FLAG (red), HP1 $\alpha$ (white), merge (blue). Arrowhead: FLAG/HP1 $\alpha$ colocalization. A") HP1 $\alpha$ localization in HEK293 cells expressing prelamin A (LA-C661M+) in combination with BAF-WT, BAF-A12T, BAF-G47E. FLAG staining (red), GFP (green), HP1 $\alpha$ (grey). Bar $10 \mu \mathrm{M}$. a") Density dot plot of FLAG and HP1 $\alpha$ colocalization staining showed in A". FLAG (red), HP1 $\alpha$ (white), merge (blue). Arrowhead and asterisk indicate FLAG/HP1 $\alpha$ colocalization. In panel (A') and (A") arrowheads indicate HP1 $\alpha$ nuclear periphery localization increase relative to untransfected cells (shown in top line); arrows: HP1 $\alpha$ intranuclear localization pattern; asterisk: partial HP1 $\alpha$ nuclear lamina localization pattern. Images shown are representative of $80 \%$ transfected cells. (B) LAP2-alpha (LAP2 $\alpha$ ) localization in HEK293 cells expressing FLAG-tagged lamin constructs in combination with GFP-BAF mutants. In the top line LAP $2 \alpha$ nuclear distribution in untransfected cells. LAP2 $\alpha$ staining (grey) is combined with DAPI (LAP2 $\alpha+$ DAPI). Bar $10 \mu \mathrm{M}$. B') LAP2 $\alpha$ localization in cells expressing wild type lamin A $(\mathrm{LA}-\mathrm{WT}+$ ) in combination with BAF-WT or BAF-A12T or BAF-G47E. FLAG staining (red), GFP (green), LAP2 $\alpha$ (grey). Bar $10 \mu \mathrm{M}$. b') Density dot plot of FLAG and LAP2 $\alpha$ colocalization staining shown in B'. FLAG (red), LAP2 $\alpha$ (grey), merge (blue). Arrowhead: FLAG/ LAP $2 \alpha$ colocalization. Arrow: absence of LAP2 $\alpha$ staining at the nuclear periphery. (B") LAP2 $\alpha$ localization in HEK293 cells expressing FLAG-tagged wild type prelamin A (LA-C661M+) BAF-WT or BAF-A12T or BAF-G47E. FLAG staining (red), GFP (green), LAP2 $\alpha$ (grey). Bar $10 \mu \mathrm{M}$. b") Density dot plot of FLAG and LAP2 $\alpha$ colocalization shown in B". FLAG (red), LAP2 $\alpha$ (white), merge (blue). Arrowhead; FLAG/LAP2 $\alpha$ colocalization. Asterisk: partial FLAG/LAP2 $\alpha$ colocalization. Arrow: absence of LAP2 $\alpha$ staining at the nuclear periphery. In (B') and (B") arrowheads indicate LAP $2 \alpha$ nuclear periphery localization, arrows indicate LAP $2 \alpha$ nucleoplasmic distribution, empty arrowheads indicate a partial LAP2 $\alpha$ nuclear lamina recruitment, asterisk indicate a more detectable LAP2 $\alpha$ nuclear lamina staining observed in LA-C661M+BAF-A12T expressing cells. Images are representative of $80 \%$ transfected cells. (C) LA-WT and LA-C661M coimmunoprecipitation experiment performed in cotransfected cells. Protein complexes recovered by anti-FLAG antibody (FLAG-IP) as well as whole lysates were subjected to FLAG, GFP, HP1 $\alpha$ and LAP2 $\alpha$ Western blotting detection. No protein bands were observed in protein $\mathrm{A} / \mathrm{G}$ negative control (A/G). 
periphery (Figure S3 asterisk and Figure 4B'-4B" and $4 b$ ' $-4 b$ " arrowheads). On the contrary, in untransfected cells or in cells expressing singularly any BAF construct, LAP2-alpha nucleoplasmic localization did not change (Figure 4B and Figure S2C). Interestingly, a decrease in prelamin A ability to modify the nuclear distribution of LAP2-alpha was observed when prelamin A constructs were expressed in combination with BAF mutants (Figure $4 B$ ' $-4 b$ ' and 4B"- $4 b$ "). The nuclear lamina recruitment of LAP2-alpha was abolished by BAF-G47E co-expression (Figure 4B'-4b' and 4B" $-4 \mathrm{~b}$ "' arrows), but it still partially occurred when cotransfected with BAF-A12T (Figure 4B empty arrowheads). In particular, we observed that the BAF-A12T inhibitory effect on LAP2-alpha nuclear periphery repositioning was more evident when LA-WT was coexpressed (Figure 4B'-4b'). Differently, LAC661M coexpression left a faint LAP2-alpha nuclear rim staining (Figure 4B"-4b" asterisks). Western blotting analysis showed that the LAP2-alpha protein level was not affected by exogenous protein expression (Figure S2C). In addition, the evaluation of fluorescence intensity profiles confirmed these results (Figure S4). A coimmunoprecipitation study performed in cotransfected cells demonstrated that BAF mutants were able to decrease prelamin A interaction with LAP2-alpha and HP1-alpha (Figure 4C). These results are in accordance with the known preferential BAF interaction with prelamin A [17], [18]; indeed, prelamin A (LA-C661M) partially affects chromatin organization (HP1-alpha and LAP2-alpha recruitment at the nuclear periphery) also when BAFprelamin A binding is compromised (prelamin A/BAFA12T complex). On the contrary, in lamin A (LA-WT) expressing cells the prelamin A protein amount seems to be insufficient, although increased, to overcome the impairment of prelamin A/AF-A12T interaction.

We confirmed our results in BAF-siRNA treated HEK293 cells expressing prelamin A constructs (Figure 5). BAF reduction (Figure 5A-5B) elicited LA-WT and LAC661M localization defects (Figure 5C arrowheads). As already observed in NGPS cells, transfected nuclei were characterized by FLAG staining rarefaction at the nuclear lamina. In accordance, the prelamin A mediated effects on HP1-alpha and LAP2-alpha nuclear localization are undetectable (in the case of HP1-alpha) (Figure 5C empty arrows) or partially detectable (in the case of LAP2-alpha) (Figure 5C). In particular, some nuclear periphery LAP2alpha aggregates were observed in LA-C661M expressing cells (Figure 5C arrow), however a complete nuclear lamina recruitment of LAP2-alpha without nucleoplasmic staining was never observed. HP1-alpha, LAP2-alpha and FLAG tagged protein amounts were unaffected by siBANF1 silencing (Figure 5A).

\section{Prelamin A-BAF interaction affects chromatin organization}

To evaluate the influence of BAF on chromatin organization due to prelamin A, we performed an electron microscopy study on HEK293 cells expressing FLAGtagged proteins in combination with BAF constructs. Untransfected cells showed a heterochromatin layer at the nuclear periphery, underlying the normally organized nuclear envelope (Figure 6 Unt.-u-u' arrows). Cells expressing LA-WT in combination with BAF-WT showed a normal chromatin organization with increase of heterochromatin clusters located at the nuclear periphery (Figure 6A-6a-6a' arrows).

On the contrary, when LA-WT was expressed in combination with BAF-A12T, the peripheral heterochromatin did not adhere to the nuclear lamina. In addition, the architecture of the nuclear wall was affected, as an aberrant duplication of the nuclear lamina and nuclear envelope was often detectable (Figure 6B-6b-6b' star/arrows).

The coexpression of LA-WT with BAF-G47E had no effect on chromatin organization. The ultrastructural appearance of HEK293 nuclei expressing this construct combination was similar to that observed in untransfected cells, even thought a mild-grade heterochromatin loss with nuclear lamina detachment was observed (Figure 6C-6c-6c' arrows).

The combination of LA-C661M with BAF-WT induced the recruitment of heterochromatin clumps at the nuclear lamina with an impressive beads-on-a-string appearance (Figure 6D-6d-6d' arrows). Interestingly, the combination of LA-C661M with BAF-A12T or BAFG47E impeded the beads-on-a-string chromatin clustering while some heterochromatin clustering was observed (Figure 6E-6e-6e'arrows and 6F-6f-6f' arrows).

\section{BAF mediates LAP2-alpha nuclear relocalization caused by progerin}

Considering that progerin, a mutated form of prelamin A accumulated in HGPS cells, interacts with BAF, we wondered if the progerin-BAF complex could affect LAP2-alpha nuclear distribution in these cells. To this aim, we first evaluated LAP2-alpha localization and expression in cells from patients affected by Hutchinson-Gilford Progeria Syndrome (Figure 7A). Using methanol fixation, we could unveil a peculiar LAP2-alpha distribution linked to progerin accumulation [27]. In control cycling cells, LAP2-alpha nucleoplasmic localization was observed (Figure 7A asterisk). In contrast, progerin increase in HGPS cells reduced intranuclear LAP2-alpha staining and 
induced a clustered nuclear lamina localization (Figure 7A arrowhead and Figure 7A') bearing perfect colocalization with progerin. LAP2-alpha nuclear redistribution was not related with its amount (Figure 7B).

For evaluating BAF involvement in progerinmediated effects on LAP2-alpha localization, we expressed FLAG-tagged progerin (FLAG- $\Delta 50$ ) in HEK293 cells (Figure S3). Endogenous BAF recruitment at the nuclear rim of FLAG- $\triangle 50$ expressing cells was observed, but aggregates were formed (Figure S3 asterisk). When GFPtagged BAF-WT was expressed in combination with FLAG$\triangle 50$ a similar distribution was observed (Figure $7 \mathrm{C}$ arrow), interestingly FLAG- $\Delta 50$ was not able to recruit the BAFG47E mutant at the nuclear lamina (Figure 7C arrowhead).
Coimmunoprecipitation studies revealed that FLAG- $\triangle 50$ was able to bind BAF-WT but failed to bind BAF-G47E mutant (Figure 7D). More interestingly, BAF-G47E was able to abrogate FLAG- $\triangle 50$ influence on LAP2-alpha nuclear localization without causing effects at the protein level (Figure 7E-7F-7G).

\section{DISCUSSION}

Chromatin disorganization is one of the main characteristics of laminopathies with prelamin A processing defects. Accumulation of different prelamin A forms highly modifies the structural and genetic organization of the nucleus [11], and pharmacological approaches reducing
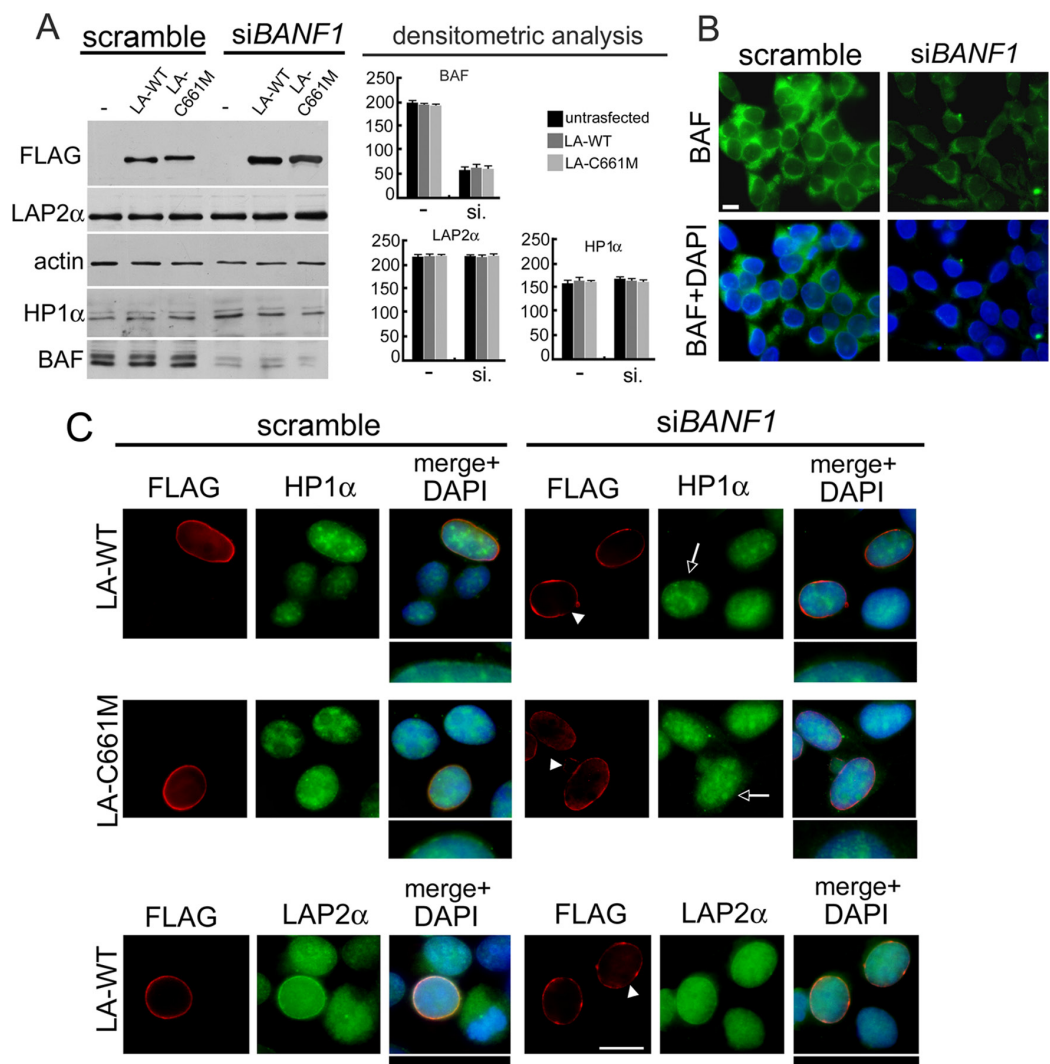

merge+
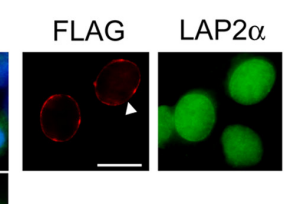

merge+
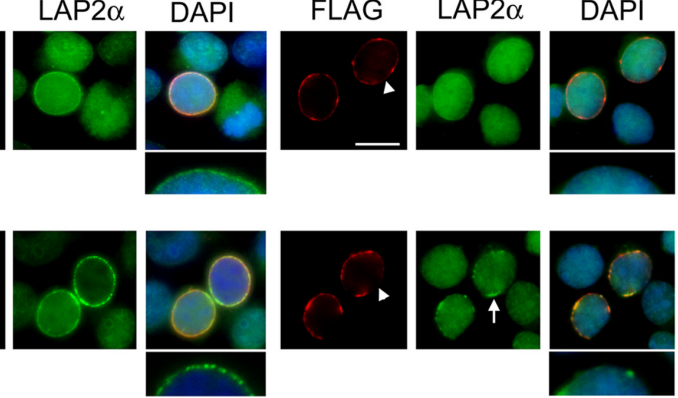

Figure 5: BAF depletion interferes with prelamin A-mediated HP1-alpha and LAP2-alpha nuclear relocalization. (A) Western blotting evaluation of total lysates from HEK293 cells subjected to BAF depletion by siRNA treatment. Cells treated with scramble or BANF1 siRNA (siBANF1) were untransfected (-) or transfected with LA-WT or LA-C661M constructs. FLAG, LAP $2 \alpha$, HP1 $\alpha$ and BAF proteins bands are shown. Actin: protein loading control. Western blotting densitometric analysis is reported. (B) Immunofluorescence evaluation of BAF in HEK293 cells treated with scramble or BANF1 siRNA. BAF (green) and DNA staining (DAPI) are shown. Bar $10 \mathrm{~mm}$. (C) Immunofluorescence detection of LA-WT, LA-C661M, HP1 $\alpha$ and LAP2 $\alpha$ in cells treated with scramble siRNA or siBANF1. FLAG (red), HP1 $\alpha$ and LAP $\alpha$ (green). DNA was costained by DAPI and shown in merge. Arrowheads: nuclear lamina rarefaction staining of FLAG tagged proteins. Empty arrows: absence of the HP1 $\alpha$ nuclear periphery staining increase. Arrow indicates the defective LAP $2 \alpha$ nuclear periphery recruitment. Higher magnification of nuclear periphery distribution of HP1 $\alpha$ and LAP $2 \alpha$ staining, merged with DAPI, is shown. Bar $10 \mu \mathrm{m}$. 
the prelamin A protein level can at least in part limit deleterious effects on nuclear functions [28-30].

Thus, the identification of prelamin A-binding proteins able to influence chromatin organization by connecting prelamin A with chromatin, is a major goal, in order to develop to intervening therapies to contrast the damage induced by prelamin A.

In this regard, we previously identified BAF as a possible prelamin A molecular mediator involved in chromatin organization defects which characterize prelamin A linked diseases [18]. In our previous study we demonstrated in vivo prelamin A-BAF interaction and, more interestingly, we observed that $\mathrm{BAF}$ was retained in the nucleus in all prelamin A-accumulating diseases [17], [18] suggesting a functional link between prelamin A and BAF. Since a human BAF proteome analysis revealed 56 high-confidence targets in a single cell type, including multiple proteins that regulate histone modifications [9] and because BAF both stabilizes the nuclear lamina structure and influences chromatin organization [19], we wondered if BAF could be a possible prelamin A modulator. The study we report here on prelamin

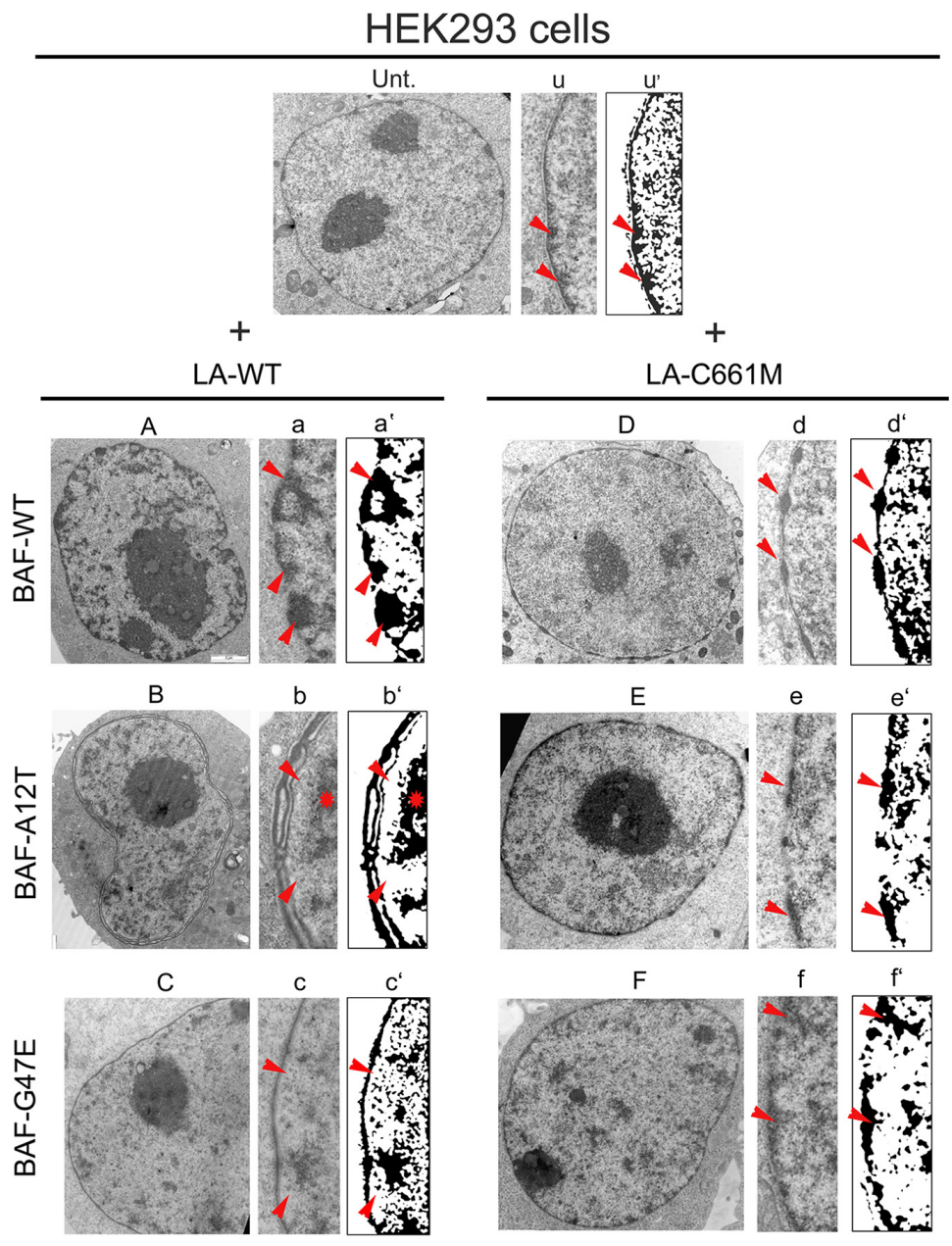

Figure 6: Electron microscopy evaluation of prelamin A/BAF complex effects on chromatin organization. Electron microscopy evaluation of HEK293 nuclei in untransfected (Unt.) or prelamin A and BAF constructs cotransfected cells. (Unt.) Ultrastructural organization of untransfected HEK293 cell nucleus. (u) Enlargement of nuclear periphery. (u') Density dot plot of picture showed in (u). Red arrowheads: peripheral heterochromatin. (A) Nucleus of cell expressing LA-WT in combination with BAF wild BAF-WT. (a) Enlargement of nuclear periphery. (a') Density dot plot of picture showed in (a). Red arrowheads: heterochromatin. (B) Nucleus of cell expressing LAWT in combination with BAF-A12T. (b) Enlargement of nuclear periphery. (b') Density dot plot of picture showed in (b). Red arrowheads: peripheral heterochromatin detachment. Red star: detached heterochromatin clump. (C) Nucleus of cell expressing LA-WT in combination with BAF-G47E. (c) Enlargement of nuclear periphery. (c') Density dot plot of picture showed in (c). Red arrowheads: heterochromatin rarefaction. (D) Nucleus of cell expressing LA-C661M in combination with BAF-WT. (d) Enlargement of nuclear periphery. (d') Density dot plot of picture showed in (d). Red arrowheads: "beads-on-a-string appearance" heterochromatin. (E) Nucleus of cell expressing LAC661M in combination with BAF-A12T. (e) Enlargement of nuclear periphery. (e') Density dot plot of picture showed in (e). Arrowheads: heterochromatin. (F) Nucleus of cell expressing LA-C661M in combination with BAF-G47E. (f) Enlargement of nuclear periphery. (f') Density dot plot of picture showed in (f). Red arrowheads: heterochromatin. Representative images of chromatin organization observed in $70 \%$ of analyzed nuclei, for each cotransfection experiment, are shown. 

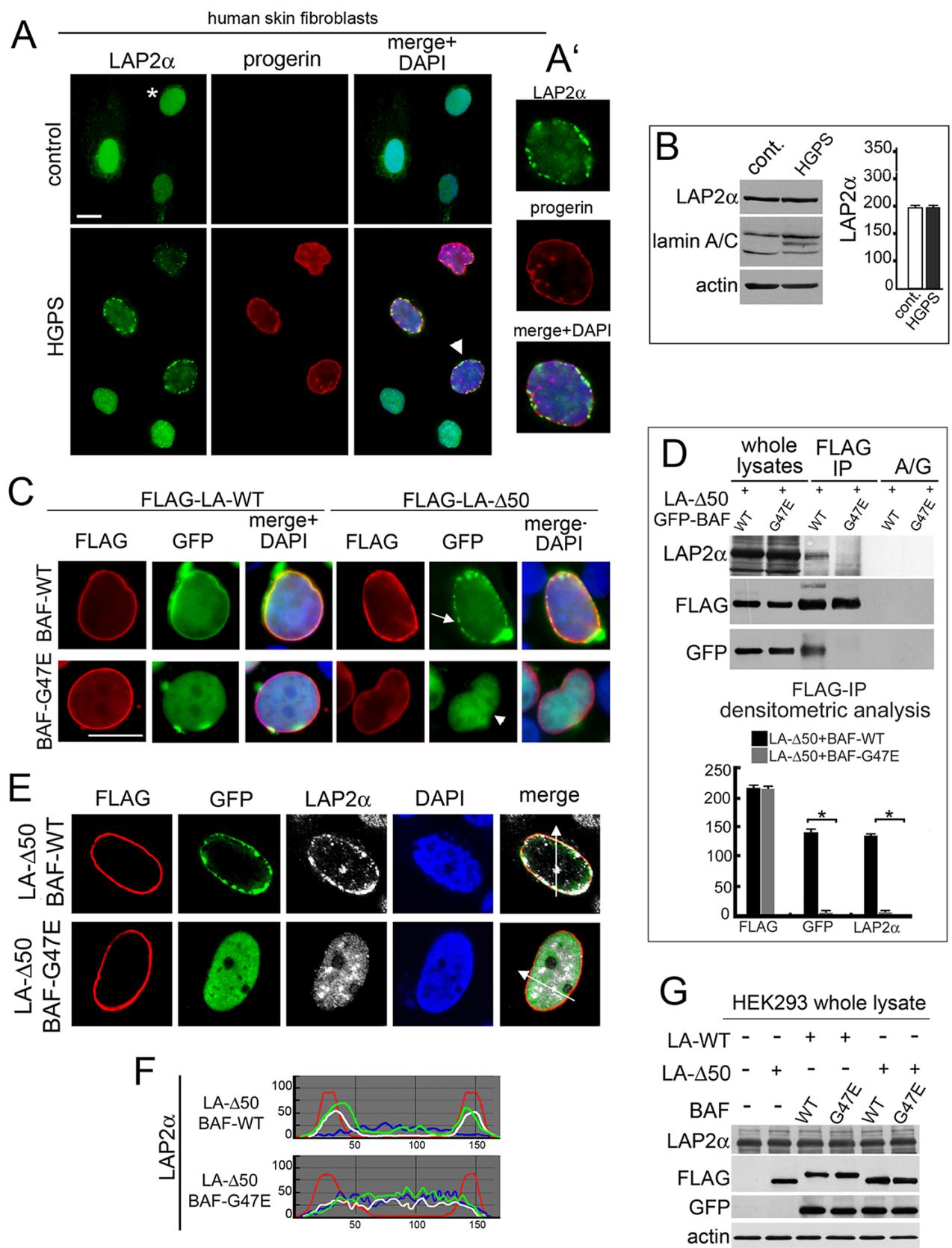

Figure 7: BAF is involved in the LAP2-alpha nuclear distribution observed in progerin expressing cells. (A) Localization of LAP $2 \alpha$ and progerin in control and HGPS cells. LAP2 $\alpha$ (green), progerin (red). DNA staining with DAPI is showed in merge. Bar $10 \mu \mathrm{m}$. Asterisk: LAP2 $\alpha$ nucleoplasmic distribution. Arrowhead: HGPS nucleus shown at higher magnification in A'. (B) Western blotting evaluation of LAP $2 \alpha$ and lamin A/C in control and HGPS cells. Actin: protein loading control. Densitometric analysis of LAP2 $\alpha$ immunoblotted bands was performed in triplicate experiments. (C) BAF-WT and BAF-G47E localization in cells expressing LA-WT or progerin construct (FLAG-LA- $\Delta 50$ ). FLAG (red), GFP (green). DNA staining with DAPI is shown in merge. Bar $10 \mu \mathrm{m}$. Arrow: BAFWT nuclear periphery aggregates. Arrowhead: BAF-G47E nucleoplasmic localization. Images are representative of $80 \%$ of transfected cells. (D) Western blotting evaluation of coimmunoprecipitation performed in cotransfected cells. FLAG, GFP and LAP2 $\alpha$ immunoblotted bands obtained from whole lysates and anti-FLAG immunoprecipitation (FLAG-IP) samples are shown. No protein bands were detected in protein A/G $(\mathrm{A} / \mathrm{G})$ negative control. Densitometric analysis of FLAG, GFP and LAP2 $\alpha$ immunoblotted bands is shown. Values are means of three independent experiments \pm S.D. $(* P<0.001)$. (E) LAP2 $\alpha$ nuclear distribution in cells coexpressing LA- $\triangle 50$ and BAF-WT or progerin unbinding mutants BAF-G47E. FLAG (red), GFP (green), LAP2 $\alpha$ (grey). DNA staining performed with DAPI is excluded in merge. Images shown are representative of $75 \%$ of cotransfected cells. (F) Fluorescence intensity profile along arrows drawn in (E). The top graph indicates intensity profile of BAF-WT (green), LA- $\Delta 50$ (red), LAP2 $\alpha$ (white) and DAPI (blue). The bottom graph indicates intensity profile of BAF-G47E (green), LA- $\Delta 50$ (red), LAP2 $\alpha$ (white) and DAPI (blue). (G) Western Blotting evaluation of LAP2 $\alpha$ in HEK293 cells untransfected or transfected with LA-WT or LA- $\Delta 50$ in combination with wild type BAF (WT) or (G47E). Immunoblotted bands obtained from detection of whole lysates are shown. LAP2 $\alpha$, FLAG, GFP. Actin: protein loading control. 
A accumulation effects on chromatin organization in NGPS cells lends evidence to this hypothesis (Figure 7). In particular we observe that A12T-mutated BAF not only affects nuclear morphology, but leads to lamin A/C localization defects and, more interestingly, interferes with the typical $\mathrm{H} 3 \mathrm{~K} 9 \mathrm{~m} 3$ intranuclear clustering induced by non-farnesylated prelamin A accumulation. In NGPS cells lamin A biogenesis is normal and mevinolin administration results in prelamin A increases both at the nuclear lamina and at intranuclear aggregates, as expected. Nevertheless, the expression of prelamin A and its colocalization with BAF-A12T and $\mathrm{H} 3 \mathrm{~K} 9 \mathrm{~m} 3$ at intranuclear aggregates appear anomalous, suggesting that the integrity of the BAF-prelamin A protein platform could be affected by the BAF-A12T mutation, which, in turn, also leads to functional impairment of prelamin A mediated chromatin organization. In accordance, we observe that though the BAF-A12T mutation does not affect BAF dimerization or BAF binding to either DNA or emerin [21], its ability to interact with lamin A and prelamin A is reduced. Thus, in NGPS cells the decrease in prelamin A capacity to recruit $\mathrm{BAF}$ in the nucleus combined with a reduction in the amount of BAF protein [21] could damage the function of the protein platform governed by prelamin A.
It has been previously described that $\mathrm{BAF}$ is necessary for proper lamin $\mathrm{A} / \mathrm{C}$ localization [31], that lamin A and prelamin A bind BAF directly [32], [10] and that lamin A and BAF influence each other's localization [33], [31]. In the absence of lamin A/C, BAF localizes exclusively in the cytoplasm and seems to be unable to translocate into the nucleus. BAF has a reciprocal influence on lamin $\mathrm{A} / \mathrm{C}$ localization. Consistent with this notion, we observed lamin $\mathrm{A} / \mathrm{C}$ honey-comb structures in NGPS cells. Therefore, it is conceivable that the decrease in both lamin $\mathrm{A} / \mathrm{BAF}$ capability to interact reciprocally and in the BAF protein level observed in NGPS cells, mimic BAF depletion causing a secondary lamin A misfunction. On the other hand, secondary lamin A defects could further worsen BAF defects in NGPS trough a feedback loop. In addition, the NGPS-BAF mutation impedes the proper localization of emerin. In NGPS cells emerin loses its nuclear distribution and is found predominantly in the cytoplasm [21]. Interestingly, emerin interacts with prelamin A in vivo [34] suggesting that the NGPS-BAF mutation could perturb the prelamin A-BAF platform by affecting the binding partners needed for proper localization of prelamin A. Indeed emerin is necessary for the intranuclear localization of prelamin A

\section{Control cell}

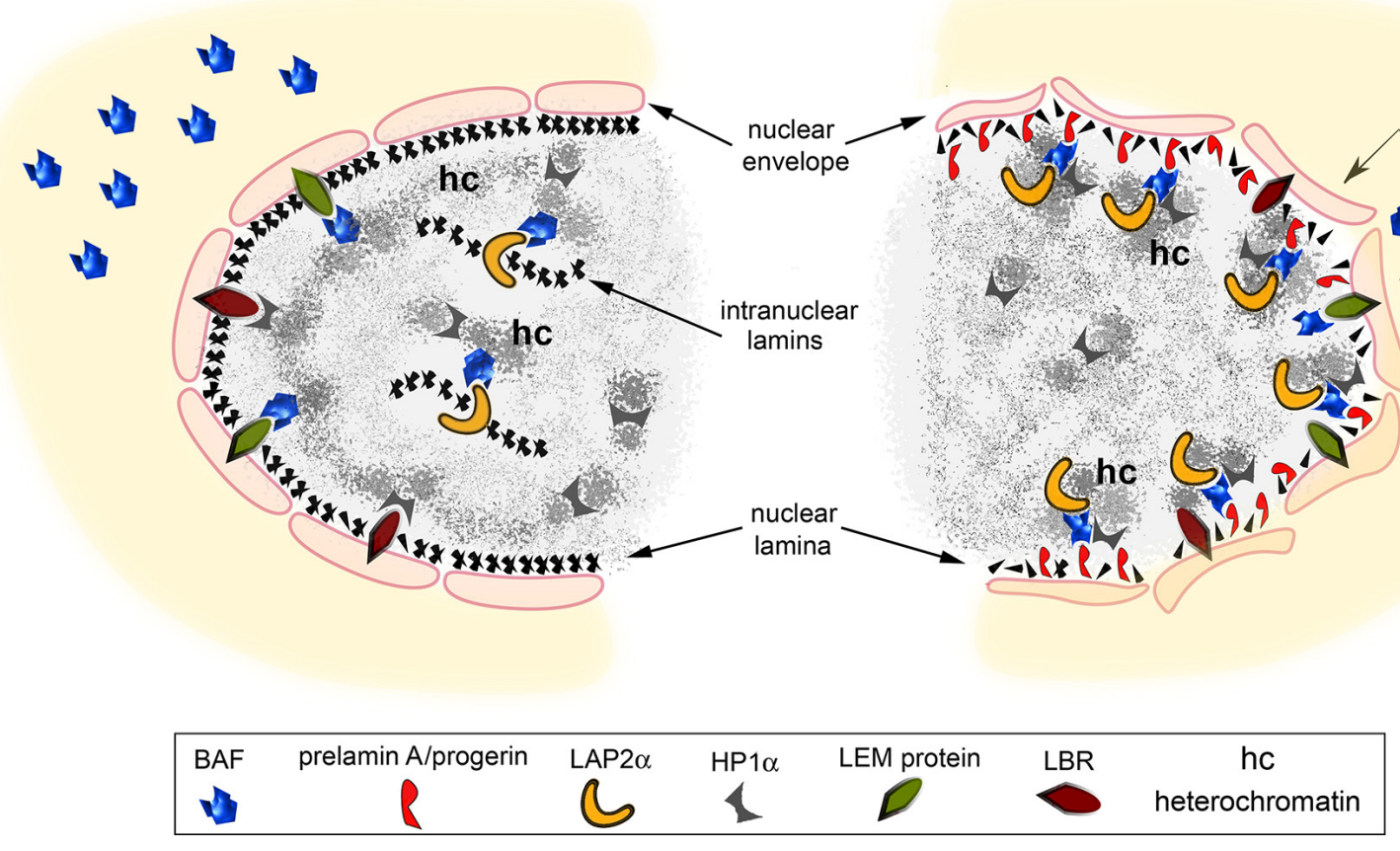

Figure 8: Speculative cartoon of the mechanism involving prelamin A-BAF interaction in chromatin organization change. In normal cells BAF localizes both at the cytoplasm and in the nucleus. At the nuclear level BAF is distributed close to the inner nuclear membrane where it interacts with LEM-proteins (LAP2, emerin and MAN1) as well as in the nucleoplasm where it binds intranuclear form of LAP2: LAP2 $\alpha$. Lamin B receptor (LBR) and heterochromatin binding protein 1 alpha (HP1 $\alpha)$ are involved in the organization of heterochromatin. The accumulation of prelamin A/progerin affects BAF localization in the cell influencing chromatin organization. In particular, the accumulation of lamin A precursors induce BAF translocation from the cytoplasm to the nuclear lamina where prelamin A and BAF interact. BAF mediates prelamin A accumulation effects on chromatin organization. Prelamin A takes advantage of BAF interaction with DNA and DNA organizing proteins to modify chromatin organization as demonstrated by LAP2 $\alpha$ and HP1 $\alpha$ nuclear relocalization. 
aggregates, as demonstrated by prelamin A mislocalization observed in emerin null cells treated to accumulate lamin A precursor [34].

The pathogenic BAF mutation leads to nuclear misshaping, increased size, lobulation and blebbing [21]. In analogy with genetic disorders due to mutations in the nuclear lamina and nuclear envelope components, we observe that such irregular nuclei are characterized by abnormalities in chromatin organization related to epigenetic changes such as trimethylation of histone $\mathrm{H} 3$ at Lys 9 and Lys27, histone $\mathrm{H} 4$ at Lys20 and the acetylation of histone H4 at Lys16 [35-37]. NGPS skin fibroblasts are characterized by loss of $\mathrm{H} 3 \mathrm{~K} 9 \mathrm{me} 3$ which becomes more evident when prelamin $\mathrm{A}$ is accumulated. The decrease of $\mathrm{H} 3 \mathrm{~K} 9 \mathrm{me} 3$ staining observed in NGPS cells is in accordance with the proposed BAF epigenetic function [9]. BAF interacts with nucleosomes and directly binds histone $\mathrm{H} 3$ and $\mathrm{H} 4$ influencing nucleosome accessibility to chromatin or, alternatively, recruiting specific regulators to chromatin [19]. In particular BAF interacts with the dimethyltransferase G9a [19]. This enzyme is responsible for H3-K9 dimethylation, a secondary histone modification necessary to silence chromatin via Suv39 h1 action. Interestingly BAF overexpression, as well as prelamin A accumulation, increases trimethylation status of histone H3 on lysine 9 [19]. In NGPS cells, such an epigenetic marker is strongly reduced and prelamin A accumulation fails to affect $\mathrm{H} 3 \mathrm{~K} 9 \mathrm{me} 3$ intranuclear localization. These findings suggest that the BAF-A12T mutation could perturb the epigenetic function of BAF affecting its ability to interact with histone $\mathrm{H} 3$ or with enzymes involved in histone $\mathrm{H} 3$ secondary modification.

We confirmed our results in NGPS cells using an experimental model. Specific markers related to chromatin organization and dynamics, HP1-alpha and LAP2-alpha [25], were evaluated under conditions of impaired prelamin A-BAF interaction. We expressed prelamin A in combination with BAF-G47E, a BAF mutant whose ability to interact with histone $\mathrm{H} 1 / \mathrm{H} 3$, inner nuclear membrane proteins (emerin and MAN1) [8] and prelamin A (this paper) is severely reduced. We observed that the expression of prelamin A constructs in combination with BAF-WT induced localization of BAF at the nuclear lamina, reorganization of HP1-alpha distribution and LAP2-alpha recruitment to the nuclear periphery, while in combination with BAF-A12T or BAF-G47E, decreased or abolished, respectively, BAF localization to the nuclear lamina as well as both HP1-alpha and LAP2-alpha relocalization in the nucleus were decreased or abolished respectively. Notably, in HGPS cells, recruitment of LAP2alpha to the nuclear periphery is mediated by WT-BAF and does not occur in the presence of BAF mutants. These findings allowed us to speculate about the possible molecular mechanism involved in progeroid syndromes linked to prelamin A processing defects and BAF mutations.
Aging is a complex process in which the most prominent hallmark is the accumulation of various types of DNA damage; among these, DSBs (DNA DoubleStrand Breaks) are the most deleterious [38, 39]. In addition, aging brings about dramatic changes in the packaging of DNA into higher-order chromatin structures [40]. Perhaps the most significant of these changes are both the global loss of highly condensed, transcriptionally silent heterochromatin [41] and the alteration in histone composition during replicative aging [42-44]. In progeroid syndromes, due to prelamin A processing impairment, heterochromatin loss and a deficiency in DNA-repair processes have been described [45], [46].

Interestingly BAF binding with proteins involved in the DNA damage response has been reported [9]. In NGPS cells, BAF mutation could interfere directly with such functions while in HGPS cells the persistence of BAFprogerin binding could impair BAF interactions or slow down the dynamics of BAF protein complexes. In this regard, it has been recently demonstrated that prelamin A accumulation affects the mobility of the BAF binding protein HP1-alpha following DNA damage, strengthening its nuclear matrix association [47]. The delay in $\gamma-\mathrm{H} 2 \mathrm{AX}$ foci formation demonstrates that the persistence of an heterochromatin status impedes DNA damage repair [47].

We observed that BAF is necessary to mediate both prelamin A and progerin effects on LAP2-alpha nuclear distribution. LAP2-alpha interacts with High Mobility Group nucleosome-binding proteins (HMGN) 5 [48]. The HMG class of proteins affects chromatin-related processes such as transcription, replication, and repair [49] causing changes in chromatin structure or at the level of histone post-translational modifications. LAP2-alpha is able to influence the nuclear distribution and mobility of HMGN5 [48], suggesting that this LEM-protein could indirectly affect the overall chromatin organization and influence the activation of specific DNA sequences.

Thus, prelamin A and progerin accumulation could also modify HMGN5 distribution across the genome through the control of LAP2-alpha nuclear localization, influencing gene expression in a pro-aging way [48].

However, further studies need to be performed in order to unveil the nuclear pathway specifically affected by the impairment of BAF function and, more interestingly, identify those common molecular events that enhance aging progression in NGPS and progeroid laminopathies.

\section{MATERIALS AND METHODS}

\section{Cell cultures, transfection and siRNA}

Skin fibroblast cultures were obtained from skin biopsies of healthy, NGPS [21] HGPS (culture passage numbers 10-15) [41] and RD (culture passage numbers 12-16) [50] patients following a written consent. Cultured were established and cultured in Dulbecco's modified 
Eagle's medium supplemented with $10 \%$ fetal calf serum (FCS) and antibiotics. For transfection experiments HEK293 cells were used. GFP-BAF and the mutated constructs BAF-A12T, BAF-G47E, full length FLAGtagged prelamin A (LA-WT, pCI mammalian expression vector) and the mutated construct LA-C661M were transiently transfected into HEK293 cells using FuGene6 reagent (Roche). The transfection efficiency was over $75 \%$. Biochemical and immunofluorescence analysis were performed $24 \mathrm{~h}$ after transfection. BAF depletion by siRNA treatment (Santa Cruz biotechnology SC-44804) was performed according to the manufacturer's protocol. In human skin fibroblast cells, the accumulation of prelamin A was obtained using $25 \mu \mathrm{M}$ mevinolin (Sigma) in growth medium for $18 \mathrm{~h}$.

\section{Western blotting and immunoprecipitation}

For Western blotting evaluation HEK293 untransfected or transfected cells were processed in lysis buffer containing $20 \mathrm{mM}$ Tris- $\mathrm{HCl}, \mathrm{pH} 7.5,1 \%$ SDS, $1 \mathrm{mM}$ Na3VO4, $1 \mathrm{mM}$ PMSF, 5\% $\beta$-mercaptoethanol and protease inhibitors. Protein were subjected to SDS gradient gel (5-20\%) electrophoresis and transferred to nitrocellulose membrane overnight at $4{ }^{\circ} \mathrm{C}$. Incubation with primary antibodies was performed for the indicated time. Bands were revealed by the Amersham ECL detection system. For coimmunoprecipitation experiments in order to evaluate tagged proteins interaction (FLAG-lamin A constructs and GFP-BAF constructs), HEK293 transfected cells were lysed in a buffer containing $50 \mathrm{mM}$ TRIS$\mathrm{HCl}, \mathrm{pH} 8.0,150 \mathrm{mM} \mathrm{NaCl}, 1 \% \mathrm{NP} 40,0.1 \% \mathrm{SDS}$ and protease inhibitors (IP-buffer). Lysates were incubated with specific antibodies overnight at $4^{\circ} \mathrm{C}$. Control immunoprecipitation were performed in the presence of aspecific immunoglobulins. After the addition of $30 \mu \mathrm{l}$ of protein A/G (Santa Cruz Biotechnology) for 45 min at $4^{\circ} \mathrm{C}$, immunoprecipitated protein complexes were washed, added to Laemmli's buffer, boiled and subjected to western blot analysis. To evaluate Lap2-alpha and Hp1-alpha interaction with lamin A/BAF protein platform a previously described methods was used [25]. Briefly, HEK 293 transfected cells were fixed in $1 \%$ formaldehyde in PBS1X for $10 \mathrm{~min}$ at room temperature, and then lysed in buffer containing 1\% SDS, $10 \mathrm{mM}$ EDTA, $50 \mathrm{mM}$ Tris- $\mathrm{HCl} \mathrm{pH} 8.0$ plus protease inhibitors. Cells were lysed by glass beads (Sigma) and thereafter by sonication. Immunoprecipitation was performed overnight at $4^{\circ} \mathrm{C}$. Immunoprecipitated protein complexes were washed and subjected to Western blot analysis.

\section{Immunofluorescence}

Human fibroblast and HEK293 transfected cells grown on coverslips were fixed in $4 \%$ paraformaldehyde at $4^{\circ} \mathrm{C}$ for $10 \mathrm{~min}$ and permeabilized with $0.15 \%$ Triton
$\mathrm{X}-100$ for $5 \mathrm{~min}$ and in methanol at $-20^{\circ} \mathrm{C}$ for 7 minutes. Samples were incubated with PBS containing 4\% BSA to saturate non-specific binding and incubated with primary antibodies and secondary antibodies. The nuclei were then counterstained with 4, 6-diamino-2-phenylindole (DAPI). The slides were mounted with an anti-fade reagent in glycerol and observed. Immunofluorescence microscopy was performed using a Nikon E600 epifluorescence microscope and a Nikon oil-immersion objective [100x magnification, 1, 3 NA (numerical aperture)]. Photographs were taken using a Nikon digital camera (DXm) and NISElement AR software. Confocal imaging was performed using a laser-scanning motorized confocal system (Nikon A1R, Nikon, Amsterdam, Netherlands) equipped with an Eclipse Ti-E inverted microscope and four laser lines $(405,488,561$, and $638 \mathrm{~nm})$. Images were acquired with a Nikon 100x PlanApo Oil 1.4 NA oil objective. For each type of quantification, laser intensities and camera settings were maintained identically within the same experiment to allow comparison of different cell expressing constructs. Localization, colocalization and mean intensity analysis were performed using NIS-Element AR. Images were processed using Adobe Photoshop (Adobe Systems).

\section{Electron microscopy}

Pellets from HEK293 transfected cells were fixed with $2.5 \%$ glutaraldehyde $0.1 \mathrm{M}$ cacodylate buffer, $\mathrm{pH} 7.3$. After post-fixation with $1 \%$ osmium tetroxide (OsO4) in cacodylate buffer for $1 \mathrm{~h}$, pellets were dehydrated in an ethanol series, infiltrated with propylene oxide and embedded in Epon resin. Ultrathin sections were stained with uranyl acetate and lead citrate (15 min each) and were observed with a Jeol Jem-1011 transmission electron microscope, operated at $100 \mathrm{kV}$. At least 100 nuclei per sample were observed.

\section{Antibodies}

The antibodies employed for western blot analysis or immunofluorescence labeling were: anti-FLAG, mouse monoclonal (Sigma M2, diluted 1:300, $1 \mathrm{~h}$, for the immunofluorescence analysis and 1:1000, $1 \mathrm{~h}$, for the Western blot analysis); anti-BAF, rabbit polyclonal (Santa Cruz Biotechnology FL-89, diluted 1:10, overnight at $4{ }^{\circ} \mathrm{C}$ for immunofluorescence analysis; anti-GFP rabbit polyclonal (Santa Cruz Biotechnology FL, diluted 1:1000 for $1 \mathrm{~h}$, for the Western blot analysis); anti-prelamin A, goat polyclonal (Santa Cruz Biotechnology SC-6214, used 1:100 overnight at $4^{\circ} \mathrm{C}$ for the immunofluorescence analysis) anti-lamin $\mathrm{A} / \mathrm{C}$, goat polyclonal (Santa Cruz Biotechnology $\mathrm{N}-18$, used $1: 100$ overnight at $4^{\circ} \mathrm{C}$, for the Western blot analysis); anti-progerin (Merck Millipore clone 13A4 diluted 1:10, overnight at $4^{\circ} \mathrm{C}$ for immunofluorescence analysis); anti-actin, goat polyclonal (Santa Cruz Biotechnology I-19, diluted 1:1000 for $1 \mathrm{~h}$, 
for the Western blot analysis); anti-trimethyl-H3K9, rabbit polyclonal (Upstate, used 1:100 for $1 \mathrm{~h}$ at room temperature for the immunofluorescence analysis); antiLAP2 $\alpha$, rabbit polyclonal Dechat [51] (used at 1:500 dilution for the immunofluorescence analysis and at 1:1000 dilution for the Western blot analysis overnight at $4^{\circ} \mathrm{C}$ ); anti-HP1 $\alpha$, rabbit polyclonal (Upstate, used 1:500 for $1 \mathrm{~h}$, for the Western blot analysis); anti-HP1 $\alpha$, rabbit polyclonal (Abcam, used at 1:100 dilution for immunofluorescence analysis).

\section{ACKNOWLEDGMENTS}

The authors wish to thank A. Valmori, S. Grasso and D. Zini for the technical assistance and William L. Blalock for the editorial assistance.

\section{GRANT SUPPORT}

A.I.Pro.Sa.B., Italian Association for Progeria, Italy A.I.D.M.E.D. Italian Association for Emery Dreifuss Muscular Distrophy

"5 per mille" 2011/2012 Rizzoli to Laboratory of Musculoskeletal Cell Biology Italian MIUR FIRB 2010 Prot. RBAP10KCNS

\section{CONFLICTS OF INTEREST}

The authors declare that they have no conflicts of interest.

\section{REFERENCES}

1. Bradley CM, Ronning DR, Ghirlando $R$, Craigie $R$, Dyda F. Structural basis for DNA bridging by barrier-toautointegration factor. Nat Struct Mol Biol. 2005; 12:935-936.

2. Margalit A, Brachner A, Gotzmann J, Foisner R, Gruenbaum Y. Barrier-to-autointegration factor-a BAFfling little protein. Trends Cell Biol. 2007; 17:202-208.

3. Segura-Totten M, Wilson KL. BAF: roles in chromatin, nuclear structure and retrovirus integration. Trends Cell Biol. 2004; 14:261-266.

4. Cai M, Huang Y, Ghirlando R, Wilson KL, Craigie R, Clore GM. Solution structure of the constant region of nuclear envelope protein LAP2 reveals two LEM-domain structures: one binds BAF and the other binds DNA. EMBO J. 2001; 20:4399-4407.

5. Lee KK, Haraguchi T, Lee RS, Koujin T, Hiraoka Y, Wilson KL. Distinct functional domains in emerin bind lamin A and DNA-bridging protein BAF. J Cell Sci. 2001; 114:4567-4573.

6. Cai M, Huang Y, Suh JY, Louis JM, Ghirlando R, Craigie R, Clore GM. Solution NMR structure of the barrier-toautointegration factor-Emerin complex. J Biol Chem. 2007; 282:14525-14535.
7. Wagner N, Krohne G. LEM-Domain proteins: new insights into lamin-interacting proteins. Int Rev Cytol. 2007; 261:1-46.

8. Montes de Oca R, Lee KK, Wilson KL. Binding of barrier to autointegration factor (BAF) to histone $\mathrm{H} 3$ and selected linker histones including H1.1. J Biol Chem. 2005; 280:42252-42262.

9. Montes de Oca R, Shoemaker CJ, Gucek M, Cole RN, Wilson KL. Barrier-to-autointegration factor proteome reveals chromatin-regulatory partners. PloS one. 2009; 4:e7050.

10. Bengtsson L, Wilson KL. Barrier-to-autointegration factor phosphorylation on Ser-4 regulates emerin binding to lamin A in vitro and emerin localization in vivo. Mol Biol Cell. 2006; 17:1154-1163.

11. Camozzi D, Capanni C, Cenni V, Mattioli E, Columbaro M, Squarzoni S, Lattanzi G. Diverse lamin-dependent mechanisms interact to control chromatin dynamics: Focus on laminopathies. Nucleus. 2014; 5.

12. Maraldi NM, Squarzoni S, Sabatelli P, Capanni C, Mattioli E, Ognibene A, Lattanzi G. Laminopathies: involvement of structural nuclear proteins in the pathogenesis of an increasing number of human diseases. J Cell Physiol. 2005; 203:319-327.

13. Driscoll MK, Albanese JL, Xiong ZM, Mailman M, Losert W, Cao K. Automated image analysis of nuclear shape: what can we learn from a prematurely aged cell? Aging (Albany NY). 2012; 4:119-132.

14. Lattanzi G, Benedetti S, Bertini E, Boriani G, Mazzanti L, Novelli G, Pasquali R, Pini A, Politano L. Laminopathies: many diseases, one gene. Report of the first Italian Meeting Course on Laminopathies. Acta Myol. 2011; 30:138-143.

15. Candelario J, Chen LY, Marjoram P, Reddy S, Comai L. A filtering strategy identifies FOXQ1 as a potential effector of lamin A dysfunction. Aging (Albany NY). 2012; 4:567-577.

16. Xiong ZM, LaDana $\mathrm{C}, \mathrm{Wu} \mathrm{D}, \mathrm{Cao} \mathrm{K}$. An inhibitory role of progerin in the gene induction network of adipocyte differentiation from iPS cells. Aging (Albany NY). 2013; $5: 288-303$

17. Capanni C, Cenni V, Haraguchi T, Squarzoni S, Schuchner S, Ogris E, Novelli G, Maraldi N, Lattanzi G. Lamin A precursor induces barrier-to-autointegration factor nuclear localization. Cell cycle. 2010; 9:2600-2610.

18. Capanni C, Squarzoni S, Cenni V, D'Apice MR, Gambineri A, Novelli G, Wehnert M, Pasquali R, Maraldi NM, Lattanzi G. Familial partial lipodystrophy, mandibuloacral dysplasia and restrictive dermopathy feature barrier-to-autointegration factor (BAF) nuclear redistribution. Cell cycle. 2012; 11:3568-3577.

19. Montes de Oca R, Andreassen PR, Wilson KL. Barrierto-Autointegration Factor influences specific histone modifications. Nucleus. 2011; 2:580-590.

20. Cabanillas R, Cadinanos J, Villameytide JA, Perez M, Longo J, Richard JM, Alvarez R, Duran NS, Illan R, Gonzalez DJ, Lopez-Otin C. Nestor-Guillermo progeria syndrome: a novel premature aging condition with 
early onset and chronic development caused by BANF1 mutations. Am J Med Genet A. 2011; 155A:2617-2625.

21. Puente XS, Quesada V, Osorio FG, Cabanillas R, Cadinanos J, Fraile JM, Ordonez GR, Puente DA, GutierrezFernandez A, Fanjul-Fernandez M, Levy N, Freije JM, Lopez-Otin C. Exome sequencing and functional analysis identifies BANF1 mutation as the cause of a hereditary progeroid syndrome. Am J Hum Gen. 2011; 88:650-656.

22. Segura-Totten M, Kowalski AK, Craigie R, Wilson KL. Barrier-to-autointegration factor: major roles in chromatin decondensation and nuclear assembly. J Cell Biol. 2002; 158:475-485.

23. Chen ZJ, Wang WP, Chen YC, Wang JY, Lin WH, Tai LA, Liou GG, Yang CS, Chi YH. Dysregulated interactions between lamin A and SUN1 induce abnormalities in the nuclear envelope and endoplasmic reticulum in progeric laminopathies. J Cell Sci. 2014; 127:1792-1804.

24. Bertacchini J, Beretti F, Cenni V, Guida M, Gibellini F, Mediani L, Marin O, Maraldi NM, de Pol A, Lattanzi G, Cocco L, Marmiroli S. The protein kinase Akt/PKB regulates both prelamin A degradation and Lmna gene expression. FASEB J. 2013; 27:2145-2155.

25. Lattanzi G, Columbaro M, Mattioli E, Cenni V, Camozzi D, Wehnert M, Santi S, Riccio M, Del Coco R, Maraldi NM, Squarzoni S, Foisner R, Capanni C. Pre-Lamin A processing is linked to heterochromatin organization. J Cell Biochem. 2007; 102:1149-1159.

26. Mattioli E, Columbaro M, Capanni C, Santi S, Maraldi NM, D'Apice MR, Novelli G, Riccio M, Squarzoni S, Foisner R, Lattanzi G. Drugs affecting prelamin A processing: effects on heterochromatin organization. Exp Cell Res. 2008; 314:453-462.

27. Pellegrini C, Columbaro M, Capanni C, D'Apice MR, Cavallo C, Murdocca M, Lattanzi G, Squarzoni S. Alltrans retinoic acid and rapamycin normalize Hutchinson Gilford progeria fibroblast phenotype. Oncotarget. 2015; 6:29914-29928. doi:10.18632/oncotarget.4939.

28. Cenni V, Capanni C, Columbaro M, Ortolani M, D'Apice MR, Novelli G, Fini M, Marmiroli S, Scarano E, Maraldi NM, Squarzoni S, Prencipe S, Lattanzi G. Autophagic degradation of farnesylated prelamin A as a therapeutic approach to lamin-linked progeria. Eur J Histochem : EJH. 2011; 55:e36.

29. Cenni V, Capanni C, Mattioli E, Columbaro M, Wehnert M, Ortolani M, Fini M, Novelli G, Bertacchini J, Maraldi NM, Marmiroli S, D'Apice MR, Prencipe S, et al. Rapamycin treatment of Mandibuloacral Dysplasia cells rescues localization of chromatin-associated proteins and cell cycle dynamics. Aging (Albany NY). 2014; 6:755-770.

30. Gordon LB, Rothman FG, Lopez-Otin C, Misteli T. Progeria: a paradigm for translational medicine. Cell. 2014; 156:400-407.

31. Haraguchi T, Koujin T, Osakada H, Kojidani T, Mori C, Masuda H, Hiraoka Y. Nuclear localization of barrier-to- autointegration factor is correlated with progression of $\mathrm{S}$ phase in human cells. J Cell Sci. 2007; 120:1967-1977.

32. Holaska JM, Lee KK, Kowalski AK, Wilson KL. Transcriptional repressor germ cell-less (GCL) and barrier to autointegration factor (BAF) compete for binding to emerin in vitro. J Biol Chem. 2003; 278:6969-6975.

33. Haraguchi T, Kojidani T, Koujin T, Shimi T, Osakada H, Mori C, Yamamoto A, Hiraoka Y. Live cell imaging and electron microscopy reveal dynamic processes of BAFdirected nuclear envelope assembly. J Cell Sci. 2008; 121:2540-2554.

34. Capanni C, Del Coco R, Mattioli E, Camozzi D, Columbaro M, Schena E, Merlini L, Squarzoni S, Maraldi NM, Lattanzi G. Emerin-prelamin A interplay in human fibroblasts. Biol Cell. 2009; 101:541-554.

35. Shumaker DK, Dechat $\mathrm{T}$, Kohlmaier A, Adam SA, Bozovsky MR, Erdos MR, Eriksson M, Goldman AE, Khuon S, Collins FS, Jenuwein T, Goldman RD. Mutant nuclear lamin A leads to progressive alterations of epigenetic control in premature aging. Proc Natl Acad Sci U S A. 2006; 103:8703-8708.

36. Krishnan V, Chow MZ, Wang Z, Zhang L, Liu B, Liu X, Zhou Z. Histone H4 lysine 16 hypoacetylation is associated with defective DNA repair and premature senescence in Zmpste24-deficient mice. Proc Natl Acad Sci U S A. 2011; 108:12325-12330.

37. Arancio W, Pizzolanti G, Genovese SI, Pitrone M, Giordano C. Epigenetic involvement in Hutchinson-Gilford progeria syndrome: a mini-review. Gerontology. 2014; 60:197-203.

38. Sedelnikova OA, Horikawa I, Redon C, Nakamura A, Zimonjic DB, Popescu NC, Bonner WM. Delayed kinetics of DNA double-strand break processing in normal and pathological aging. Aging cell. 2008; 7:89-100.

39. Sedelnikova OA, Horikawa I, Zimonjic DB, Popescu NC, Bonner WM, Barrett JC. Senescing human cells and ageing mice accumulate DNA lesions with unrepairable doublestrand breaks. Nat Cell Biol. 2004; 6:168-170.

40. Lopez-Otin C, Blasco MA, Partridge L, Serrano M, Kroemer G. The hallmarks of aging. Cell. 2013; 153:1194-1217.

41. Lattanzi G, Ortolani M, Columbaro M, Prencipe S, Mattioli E, Lanzarini C, Maraldi NM, Cenni V, Garagnani P, Salvioli S, Storci G, Bonafe M, Capanni C, et al. Lamins are rapamycin targets that impact human longevity: a study in centenarians. J Cell Sci. 2014; 127:147-157.

42. Feser J, Truong D, Das C, Carson JJ, Kieft J, Harkness T, Tyler JK. Elevated histone expression promotes life span extension. Mol Cell. 2010; 39:724-735.

43. O'Sullivan RJ, Kubicek S, Schreiber SL, Karlseder J. Reduced histone biosynthesis and chromatin changes arising from a damage signal at telomeres. Nat Struct Mol Biol. 2010; 17:1218-1225.

44. Tsurumi A, Li WX. Global heterochromatin loss: a unifying theory of aging? Epigenetics. 2012; 7:680-688. 
45. McCord RP, Nazario-Toole A, Zhang H, Chines PS, Zhan Y, Erdos MR, Collins FS, Dekker J, Cao K. Correlated alterations in genome organization, histone methylation, and DNA-lamin A/C interactions in Hutchinson-Gilford progeria syndrome. Genome Res. 2013; 23:260-269.

46. Misteli T, Scaffidi P. Genome instability in progeria: when repair gets old. Nat Med. 2005; 11:718-719.

47. Liu J, Yin X, Liu B, Zheng H, Zhou G, Gong L, Li M, Li X, Wang Y, Hu J, Krishnan V, Zhou Z, Wang Z. HP1alpha mediates defective heterochromatin repair and accelerates senescence in Zmpste24-deficient cells. Cell cycle. 2014; 13:1237-1247.

48. Zhang S, Schones DE, Malicet C, Rochman M, Zhou M, Foisner R, Bustin M. High mobility group protein N5 (HMGN5) and lamina-associated polypeptide 2alpha
(LAP2alpha) interact and reciprocally affect their genome-wide chromatin organization. J Biol Chem. 2013; 288:18104-18109.

49. Reeves R. Nuclear functions of the HMG proteins. Biochimica et biophysica acta. 2010; 1799:3-14.

50. Columbaro M, Mattioli E, Schena E, Capanni C, Cenni V, Levy N, Navarro CL, Del Coco R, Squarzoni S, Camozzi D, Hutchison CJ, Wehnert M, Lattanzi G, et al. processing and functional effects in restrictive dermopathy. Cell cycle. 2010; 9:4766-4768.

51. Dechat T, Gajewski A, Korbei B, Gerlich D, Daigle N, Haraguchi T, Furukawa K, Ellenberg J, Foisner R. LAP2alpha and BAF transiently localize to telomeres and specific regions on chromatin during nuclear assembly. J Cell Sci. 2004; 117:6117-6128. 